\title{
L'itinérance comme vecteur de reproduction des rapports de genre inégalitaires: une analyse des relations intimes chez les jeunes
}

\author{
CÔTÉ, Philippe-Benoit \\ FLYNN, Catherine \\ BLAIS, Martin \\ MANSEAU, Hélène \\ FOURNIER, Émilie
}

\section{RÉSUMÉ}

Cette étude vise à comprendre les différences de genre au sein des relations intimes chez les jeunes en situation d'itinérance. À partir d'une méthode qualitative, une analyse différenciée selon les sexes (ADS) de 32 entrevues réalisées auprès de jeunes en situation d'itinérance (18 femmes, 14 hommes) a été effectuée. L'analyse montre que les jeunes hommes et les jeunes femmes font une expérience distincte des relations intimes en raison des rapports de genre inégalitaires qui caractérisent la situation d'itinérance. Il est révélé que les jeunes femmes, pour assurer leur sécurité, se résignent à accepter la violence exercée par leur partenaire intime, tandis que les jeunes hommes se désinvestissent des relations intimes au profit de la quête d'un statut privilégié. Cette étude montre que l'itinérance constitue un vecteur de reproduction des rôles de genre traditionnels venant amplifier les rapports inégalitaires au sein des relations intimes chez les jeunes.

Mots-clés : itinérance, intimité, genre, analyse différenciée selon les sexes, rapports sociaux inégalitaires.

\begin{abstract}
This study aims to understand the gender differences in intimate relationships among homeless youth. A qualitative analysis was carried out on the basis of individual interviews with 32 homeless youth (18 women, 14 men). The results show that young men and women do not experience intimate relationships in the same way because of the unequal gender relations that characterize the homelessness context. It is revealed that young women, in order to ensure their safety, resign themselves to accept intimate partner violence, while young men keep their intimate relationships involvement minimal in favor of the quest for a privileged status. This study highlights that the homelessness context constitutes a vector of reproduction of traditional gender roles that amplify the inequalities in the intimate relationships among homeless youth.
\end{abstract}

Key words: homelessness, intimacy, gender, gender-based analysis, unequal social relations. 


\section{INTRODUCTION}

Certains chercheurs avancent que la précarité économique vécue par les jeunes en situation d'itinérance les conduirait à se replier sur des rôles de genre traditionnels (Lanzarini, 2000 ; Laporte et al., 2007 ; Osthus et Sewpaul, 2014). Le concept de « rôle de genre » réfère aux rôles que la société attribue aux hommes et aux femmes sur une base différentielle en fonction des représentations sociales de la masculinité et de la féminité (Tolman, Striepe et Harmon, 2003). De façon générale, les rôles de genre traditionnels qui caractérisent la masculinité renvoient, entre autres, à l'indépendance, à la capacité de pourvoir aux besoins de la famille, au contrôle des émotions, à la séduction et à l'agressivité (Mahalik et al., 2003), tandis que ceux qui évoquent la féminité comprennent notamment la dépendance, la gentillesse, le romantisme, la fidélité sexuelle et l'assujettissement (Mahalik et al., 2005). Selon Tolman et al. (2003), ces rôles de genre sont produits et structurés par le système patriarcal et, plus spécifiquement, par l'institutionnalisation de l'hétérosexualité qui crée des rapports inégalitaires entre les hommes et les femmes.

En contexte de précarité, l'adhésion à ces rôles de genre pousserait les jeunes à mobiliser des stratégies de débrouillardise différenciées selon le genre. Ainsi, les jeunes hommes en situation d'itinérance déploient surtout des stratégies de débrouillardise axées sur la criminalité, telles que la vente de drogues (Gwadz et al., 2007), qui leur permettent d'obtenir des ressources financières et matérielles nécessaires à leur survie (Bellot, 2003 ; O'Grady et Gaetz, 2004 ; Côté et al., 2013). L'accès à ces ressources les rend plus attirants auprès des jeunes femmes qui cherchent à subvenir à leurs besoins de subsistance et à se protéger de la violence associée aux conditions de vie précaires et instables de la situation d'itinérance (Lanzarini, 2000 ; Jamoulle, 2005 ; Laporte et al., 2007). Selon Lanzarini (2000), l'adhésion des jeunes hommes aux rôles de genre traditionnels les pousserait à trouver une source de valorisation dans le fait de protéger les jeunes femmes des dangers extérieurs. Ce repli sur les rôles de genre traditionnels entraîne les jeunes hommes à adopter des attitudes misogynes et des comportements violents à l'égard des jeunes femmes (Lanzarini, 2000 ; Osthus et Sewpaul, 2014). D'ailleurs, si les jeunes hommes en situation d'itinérance vivent davantage d'agressions physiques, les jeunes femmes, elles, font davantage l'expérience d'agressions sexuelles (Cauce et al., 2000). Cependant, certains jeunes hommes évitent d'établir des relations intimes dans le contexte de l'itinérance lorsqu'ils ne parviennent pas à obtenir les ressources nécessaires pour assumer leur rôle de pourvoyeur et de protecteur (Laporte et al., 2007 ; Kennedy et al., 2013).

En ce qui concerne les jeunes femmes, elles organisent une grande partie de leur quotidien à se prémunir contre les violences physiques et sexuelles en mobilisant différentes stratégies : se procurer un chien (Jamoulle, 2009 ; Flynn, Damant et Lessard, 2015), adopter une attitude résistante (Huey et Berndt, 2008), établir des relations intimes homosexuelles (Bellot, 2003), avoir recours à un proxénète dans le cadre de la prostitution (O'Grady et Gaetz, 2004) ou s'unir à un homme pour en tirer une forme de protection (Blais et al., 2012 ; Jamoulle, 2009 ; Lanzarini, 2000 ; Watson, 2011 ; Osthus et Sewpaul, 2014). Les relations intimes permettent aux jeunes femmes d'obtenir une sécurité émotionnelle, physique et matérielle (Kirst, Erickson et Strike, 2009; Watson, 2013) et d'améliorer leurs conditions de vie précaires et instables associées à l'itinérance (Laporte et al., 2007). Par contre, l'étude ethnographique de Stablein (2011) montre que les rapports entre les jeunes hommes et les jeunes femmes en situation d'itinérance se déploient sous l'angle de l'exploitation, principalement sur le plan sexuel. On constate ainsi que les jeunes femmes sont fréquemment victimes d'agressions sexuelles de la part des hommes qui leur offrent un hébergement ou avec lesquels elles forment un 
couple (Watson, 2011). Malgré le fait que la prostitution constitue une stratégie de débrouillardise principalement utilisée par les jeunes femmes en situation d'itinérance (Roy et al., 2000), la différence de prévalence entre les genres disparaît lorsque la consommation de substances est considérée comme le principal motif à cette pratique sexuelle (Walls et Bell, 2011).

Ces travaux éclairent une construction genrée des relations intimes chez les jeunes en situation d'itinérance, mais comportent des lacunes théoriques et méthodologiques auxquelles le présent article veut tenter de répondre. Premièrement, en négligeant l'influence du système patriarcal, ces études occultent les rapports de pouvoir économico-sexuels qui traversent les relations intimes. Le concept “d'échange économico-sexuel » (Tabet, 2004) présente les relations hétérosexuelles comme un système d'oppression et de domination au sein duquel les femmes fournissent des services sexuels pour obtenir différentes formes de compensations de la part des hommes. Ce concept permet de repositionner les relations intimes à l'aune du système patriarcal et de capter les rapports de privilège et d'oppression entre les hommes et les femmes. Deuxièmement, cette conceptualisation par les stratégies de débrouillardise laisse présumer que les jeunes en situation d'itinérance exploitent leurs partenaires intimes (Lanzarini, 2000 ; Pichon, 2007), tout en minimisant les fonctions affectives, émotionnelles et identitaires des relations intimes contemporaines (Giddens, 1992 ; Bauman, 2004). Pourtant, l'intimité constitue un espace partagé par les partenaires qui permet une valorisation et une reconnaissance nécessaires à la définition de soi (Giddens, 1992 ; Beck et Beck-Gernsheim, 1995). Troisièmement, ces travaux s'appuient principalement sur des échantillons non mixtes, ce qui limite la capacité d'analyse différenciée par sexe au sein d'une même étude. Une description des expériences selon le sexe permet d'éviter de produire une lecture homogène des phénomènes et de tenir compte de l'unicité des expériences des femmes et des hommes (Secrétariat à la condition féminine, 2007).

C'est dans ce contexte que cet article propose une analyse différenciée selon les sexes (ADS) des relations intimes chez les jeunes en situation d'itinérance. L'ADS a été développée par le gouvernement du Québec en 1997 dans le but de permettre aux gestionnaires d'adopter des politiques, programmes, mesures et services mieux adaptés selon les genres et, ultimement, de parvenir à une société plus égalitaire entre les femmes et les hommes (Massé, Laberge et Massé, 2002 ; Secrétariat à la condition féminine, 2007 ; Ministère de la Santé et des Services sociaux, 2011). II s'agit d'un outil permettant d'identifier la construction et l'influence des rapports de pouvoir présents entre les femmes et les hommes au sein de situations de vie spécifiques, telles que l'itinérance. À l'étape de l'analyse des données, l'ADS permet d'identifier les similitudes et les distinctions entre les hommes et les femmes et de documenter les rapports de genre inégalitaires qui y sont sous-jacents (Massé et al., 2002). Inscrit dans un cadre d'analyse basé sur les rapports de pouvoir, l'objectif de cet article est de documenter les différences de genre quant à l'expérience que font les jeunes en situation d'itinérance des relations intimes.

\section{MÉTHODOLOGIE}

À partir d'une ADS, cet article présente une analyse secondaire des témoignages de 32 participants rencontrés dans le cadre d'une étude sur les parcours amoureux et sexuels des jeunes en situation d'itinérance (voir notamment Blais et al., 2012 ; Côté et al., 2013 ; Côté et al., 2016). Les résultats de cette étude montraient une différenciation genrée de leurs expériences amoureuses et sexuelles, sans toutefois en offrir une conceptualisation théorique, limite à laquelle remédie la présente analyse. Le recrutement des participants a été réalisé d'octobre 2007 à avril 2010 à partir de deux stratégies. 
Premièrement, des encarts publicitaires ont été affichés au sein de différentes ressources pour jeunes en situation d'itinérance à Montréal afin de publiciser le projet de recherche. Deuxièmement, les jeunes rencontrés ont été invités à transmettre la proposition de recrutement à leurs amis et connaissances en situation d'itinérance. L'admissibilité à l'étude reposait sur trois critères : 1) être âgé de 18 à 25 ans ; 2) avoir été sans endroit où dormir au moins une fois durant la dernière année ; et 3) avoir fréquenté à plusieurs reprises des ressources pour jeunes en situation d'itinérance durant la dernière année.

Au total, 32 jeunes en situation d'itinérance ( 18 femmes, 14 hommes) âgés de 18 à 27 ans (moyenne de 22 ans) ont été rencontrés. Malgré le critère de l'âge, un jeune de plus de 25 ans (Benoît, âgé de 27 ans) a été inclus dans l'échantillon final. Comme le recrutement s'est élaboré selon l'échantillonnage théorique, c'est-à-dire qu'il s'est construit par étapes successives en réponse aux analyses des données et des lectures théoriques (Laperrière, 1997), le récit de Benoît s'est ajouté en raison de sa pertinence empirique. Une entrevue semi-dirigée d'environ une heure a été réalisée avec les participants à partir des dimensions suivantes: 1) les représentations de l'amour et de la sexualité ; 2) les expériences amoureuses et sexuelles vécues ; 3) les représentations de la situation d'itinérance ; 4) les expériences vécues en situation d'itinérance ; 5) les relations interpersonnelles en situation d'itinérance ; et 6) les représentations de l'avenir. Cette étude a reçu l'approbation éthique du Comité institutionnel d'éthique de la recherche de l'Université du Québec à Montréal. Le consentement libre et éclairé des jeunes a été assuré à l'aide d'un formulaire de consentement qui a été lu, discuté et signé par chacun des participants. Tous les noms ont été remplacés dans les retranscriptions par des prénoms fictifs. Un montant de $30 \$$ a été remis à chacun des participants à titre de dédommagement pour leur déplacement.

L'analyse qualitative des données s'est inspirée des étapes de décontextualisation et de recontextualisation des données proposées par Tesch (1990). Pour l'étape de la décontextualisation, le matériel recueilli a fait l'objet d'une codification exhaustive, phrase par phrase, afin d'identifier systématiquement les thèmes et les unités de sens de chacun des témoignages. Pour l'étape de la recontextualisation, les unités de sens ont été regroupées sur la base de leur proximité symbolique pour former des catégories conceptuelles, c'est-à-dire des descriptions analytiques succinctes visant à désigner le plus fidèlement possible l'orientation générale des messages livrés par les participants. C'est à l'étape de la recontextualisation que l'ADS a été mobilisée pour faire émerger des distinctions ou des similitudes dans la construction des relations intimes chez les jeunes femmes et les jeunes hommes en situation d'itinérance. À cette étape, les dimensions de la survie, de la prostitution et de la violence au sein des relations intimes ont été particulièrement ciblées. Afin de s'assurer de la fiabilité du processus d'analyse de données, la codification des entrevues et l'élaboration des catégories conceptuelles ont été validées, sous forme de fidélisation interjuge, par tous les auteurs de l'article.

II importe de tenir compte de certaines limites de cette étude, notamment celles associées à la taille réduite de l'échantillon. Étant donné que seulement 32 témoignages ont été analysés pour cet article, les résultats dégagés ne peuvent être généralisés à l'ensemble de la population des jeunes en situation d'itinérance. Également, devant le malaise de certains jeunes à discuter de leurs relations affectives et sexuelles, les intervieweurs ont dû adopter parfois une attitude plus directive afin de relancer leur discours. II est possible que les jeunes rencontrés aient produit un discours adapté aux demandes implicites des chercheurs. II est donc important de considérer cet article comme une étude exploratoire. 


\section{RÉSUltats}

Les témoignages des participants révèlent que les relations intimes des jeunes, autant des femmes que des hommes, sont vécues avec beaucoup de difficultés, mais qu'elles sont paradoxalement considérées comme importantes dans le contexte de l'itinérance. Par ailleurs, l'ADS montre que les jeunes femmes et les jeunes hommes font une expérience distincte des relations intimes, sur la base notamment des rapports de genre inégalitaires qui sont exacerbés dans le contexte de l'itinérance. Les prochaines sections décrivent les points de convergence et de divergence quant à l'expérience des relations intimes chez les jeunes femmes et les jeunes hommes en situation d'itinérance.

\section{Point de convergence : des relations intimes difficiles, mais importantes dans le contexte de l'itinérance}

Tant les jeunes femmes que les jeunes hommes indiquent que les conditions de vie difficiles de la situation d'itinérance font obstacle au développement et au maintien de relations intimes. La précarité économique et l'instabilité résidentielle auxquelles les jeunes sont exposés dans le contexte de l'itinérance les poussent à se concentrer sur eux-mêmes pour répondre à leurs besoins de subsistance, tels que se nourrir, se loger et se vêtir, ce qui fait en sorte qu'ils n'ont pas l'énergie ni le temps pour s'investir dans une relation intime. Un des jeunes résume bien la situation :

Quand j'étais dans rue, mon cerveau tournait à 100 milles à l'heure, il fallait tout le temps que je pense à tout ! Je n'ai pas de cigarette, je n'ai pas d'argent, je n'ai pas de bouffe, je n'ai pas de toit sur ma tête... Qu'est-ce que je fais ? Je m'en vais où ? Je n'ai pas le temps de m'occuper d'une fille en plus. (André, 22 ans)

Les jeunes rapportent également que la consommation de drogue complique, voire limite leur engagement dans une relation intime. Ils témoignent d'expériences où la consommation de drogue a pris, pour eux-mêmes ou leur partenaire, plus d'importance que la relation elle-même, en raison notamment de la dépendance qu'elle a engendrée. Ainsi, la consommation de drogue semble devenir une « expérience totale » (Castel, 1998), qui réorganise la vie des jeunes autour d'elle et relègue au second plan toute autre expérience. Dans certains cas, la dépendance à la drogue est telle qu'elle conduit certains jeunes à rompre avec une expérience amoureuse et familiale qu'ils considéraient pourtant comme très importante.

J'ai recommencé à consommer après avoir accouché de ma fille. Et la drogue a pris euh... plus de place dans ma vie. J'ai choisi la drogue au lieu de ma fille, de ma famille, dans le fond... Parce que j'avais le goût de tripper, c'est tout. (Lucie, 25 ans)

Au-delà de ces obstacles, les jeunes témoignent de l'importance des relations intimes pour surmonter les conditions de vie précaires et instables de leur situation d'itinérance. Ils mentionnent que leurs partenaires intimes jouent un rôle prépondérant dans leur expérience d'itinérance par l'établissement d'un lien de collaboration leur permettant de répondre à leurs besoins de survie. Ils décrivent leurs partenaires intimes comme des "partenaires de rue » avec lesquels ils unissent leurs stratégies de débrouillardise pour partager leurs gains économiques et matériels. Les relations intimes constituent donc une source importante de soutien matériel et affectif qui leur permet de conjuguer avec les difficultés associées à l'itinérance, comme la précarité économique, l'instabilité résidentielle, la stigmatisation et la discrimination. 
Mon chum, quand il quête avec moi, c'est lui qui demande [de l'argent] au monde. Des fois, si on a un montant à faire, par exemple 20 \$ ou 40 \$, on peut se séparer et on fait la moitié chaque. Comme ça, ça va plus vite... Ça aide et, en plus, on se tient compagnie. (Émilie, 18 ans)

Si les jeunes hommes et les jeunes femmes décrivent l'intimité comme une expérience complexe, mais nécessaire à leur subsistance dans le contexte de l'itinérance, la nature et le sens de ces relations intimes diffèrent selon le genre.

\section{Les jeunes femmes en situation d'itinérance: des relations intimes considérées comme nécessaires pour leur sécurité et leur survie}

En raison des dangers et des menaces auxquels les jeunes femmes sont exposées en situation d'itinérance, les relations intimes sont souvent considérées comme une stratégie nécessaire pour assurer leur sécurité et leur survie. Si plusieurs jeunes femmes rapportent vivre des expériences de violence de la part de leur partenaire intime, elles disent toutefois s'y résigner pour se protéger des conditions de vie difficiles et dangereuses de l'itinérance. Pour tenter d'améliorer leur situation, certaines jeunes femmes vont jusqu'à mobiliser des activités prostitutionnelles comme stratégie de dernier recours.

Une résignation à l'égard de la violence dans leurs relations intimes pour assurer leur sécurité

Plusieurs jeunes femmes disent ressentir beaucoup d'anxiété et d'inquiétude en raison du caractère dangereux et violent qu'elles associent à la situation d'itinérance, notamment les risques d'homicide et d'agressions sexuelles. Pour prévenir ces dangers, les jeunes femmes disent avoir recours à différentes stratégies de débrouillardise, dont la création de relations intimes pour se sentir protégées par leurs partenaires. La représentation des partenaires intimes masculins comme source de sécurité est d'une telle importance chez certaines femmes qu'elles disent s'assurer de toujours établir une relation intime afin de ne pas être seules dans le contexte de l'itinérance.

Quand je me ramasse dans la rue, les relations [intimes] ont été une protection quand je me couchais le soir dans la rue, pour ne pas que je me fasse attaquer ou violer... J'ai toujours eu des relations dans la rue pour ne pas être toute seule quand je dormais. (Amélie, 20 ans)

Malgré ce sentiment de protection associé aux relations intimes, les deux tiers des participantes $(12 / 18)$ mentionnent avoir été victimes de violence de la part de leurs partenaires intimes. Ces expériences de violence prennent différentes formes : psychologique (ex. : manipulation, contrôle, jalousie), verbale (ex. : menaces, insultes, manque de respect) et physique (ex. : secouer, pousser, frapper). Les participantes disent toutefois se résigner à accepter cette violence au profit d'une relation qui leur permet d'assurer leur sécurité.

[Mon chum] jouait beaucoup avec mes sentiments, surtout au niveau de mon passé. J'étais une salope pour lui. C'était de la manipulation verbale, mais violente en même temps [...] Un moment donné, il a été violent envers moi, physiquement... Mais, finalement, je revenais avec lui et je me laissais battre. Je me laissais battre. (Jessica, 20 ans)

L'utilisation de la prostitution comme stratégie de dernier recours afin d'assurer leur survie

Pour améliorer leurs conditions de vie ou même assurer leur survie, certaines jeunes femmes disent 
mobiliser la prostitution. Ces pratiques prostitutionnelles prennent la forme soit d'une monnaie d'échange pour obtenir un hébergement ou de la nourriture, soit d'un travail rémunéré (ex. : escorte, prostitution de rue, danse érotique) pour obtenir de l'argent leur permettant de se procurer des biens nécessaires à leur survie. La prostitution est considérée comme une stratégie de dernier recours quand elles ne parviennent pas à identifier d'autres moyens pour assurer leur subsistance. Lorsque les jeunes femmes découvrent d'autres stratégies de débrouillardise, comme les ressources d'hébergement ou l'aide sociale, la prostitution est délaissée au profit d'une autre solution jugée moins dénigrante. C'est ce dont témoigne Allie, par exemple :

Je ne savais pas qu'il y avait un bien-être social, je ne savais pas que le gouvernement payait et j'étais vraiment en mauvaise santé. Mais des fois, je ne savais pas où j'allais coucher et comme je ne voulais pas dormir dans la rue, alors je cherchais quelqu'un avec qui je pouvais m'en aller. À la fin, je finissais par coucher avec cette personne-là. Mais je n'avais pas envie. (Allie, 21 ans)

\section{Les jeunes hommes en situation d'itinérance : des relations intimes désinvesties au profit d'un statut privilégié}

Pour ce qui est des jeunes hommes en situation d'itinérance, ils se désinvestissent, soit partiellement, soit totalement des relations intimes au profit de la quête d'un statut privilégié. Certains d'entre eux disent se retirer de toutes formes de relations intimes en raison d'un sentiment d'humiliation suscité par leur situation d'itinérance. Pour leur part, d'autres jeunes hommes disent exploiter leur position sociale associée à la criminalité pour exhorter les jeunes femmes à échanger des faveurs sexuelles.

\section{Un retrait des relations intimes en raison de l'humiliation associée à leur situation}

Certains jeunes hommes mentionnent faire l'expérience d'un sentiment d'humiliation provoqué par la précarité et l'instabilité de la situation d'itinérance. En réaction à ce sentiment, ces jeunes hommes disent se retirer de toutes formes de relations intimes en situation d'itinérance. Ils rapportent que I'humiliation qui y est associée les empêche de se percevoir comme des hommes attirants et séduisants. Ces jeunes préfèrent alors s'abstenir de tout contact avec des partenaires intimes par crainte qu'elles leur renvoient une image humiliante d'eux-mêmes, à savoir celle d'hommes pauvres, sans logement et sans emploi.

Tu ne commences pas une relation quand tu es dans une place de même [une maison d'hébergement], parce que c'est comme une honte. Ne pas avoir de logement et être dans la rue, comme je le suis en ce moment, puis d'être mal pris, j'aurais honte d'amener une copine ici... J'aurais honte de l'amener ici, parce que j'ai honte moi-même d'être ici, de ne pas être dans un logement. (Alex, 22 ans)

Plutôt que de s'investir dans les relations intimes, ces jeunes hommes disent concentrer leur attention sur la sortie de l'itinérance. Selon eux, le fait de développer et d'entretenir des relations intimes les ancrerait davantage dans cette situation plutôt que de les aider à s'en sortir. L'amour et la famille sont alors vus comme des objectifs à long terme, au moment où leurs conditions de vie seront améliorées, ce qui, selon leurs dires, leur permettrait de chasser l'humiliation associée à la situation d'itinérance.

Je veux me sortir de là le plus tôt possible. C'est pour ça que ces temps-ci, les femmes, je ne fais que les regarder et rêver. [...] II y en a plein des belles filles, sauf qu'elles ne sont pas pour moi... Elles 
sont dans les branches de la consommation et de la quête. Et moi, je veux décrocher du monde de la rue, je n'aime pas ça. (Christian, 24 ans)

Un investissement dans les activités criminelles qui les rend attirants auprès de partenaires intimes

D'autres jeunes hommes disent mobiliser différentes activités criminelles, dont la vente de drogues, pour contrer les conditions de vie précaires et instables de la situation d'itinérance. Ces activités criminelles leur donnent accès à des ressources financières et matérielles qui leur confèrent un certain prestige, leur permettant de se démarquer des autres jeunes en situation d'itinérance. L'intégration au milieu criminel leur procure non seulement une certaine autonomie économique, mais aussi une image sociale de réussite qui les place dans une position de domination à l'égard des autres jeunes en situation d'itinérance.

J'étais "boss de rue ", c'est moi qui fournissais les vendeurs de drogues. On pouvait dire que j'étais devenu quelqu'un! Quand je me promenais sur la rue, le monde me reconnaissait. Quand j'entrais dans le parc, le monde savait qui j'étais et que c'était mon parc. (Sébastien, 19 ans)

Ces jeunes hommes indiquent que l'aura de la criminalité les rend attirants auprès des jeunes femmes en situation d'itinérance, puisque ces dernières souhaitent bénéficier de leurs ressources pour obtenir de la nourriture, des vêtements ou un hébergement. Ils disent utiliser leur statut socio-économique privilégié en situation d'itinérance pour inciter les jeunes femmes à échanger, de manière explicite ou implicite, des faveurs sexuelles. Ces relations sexuelles leur permettent donc de projeter une image sociale de performance et, par conséquent, d'accroître leur sentiment de réussite sociale en situation d'itinérance.

Je rencontrais des filles un peu partout, elles étaient contentes du service [de vente de drogues] que je leur avais offert... Je leur demandais si elles voulaient faire un petit tour chez mon ami avec moi. Alors, on fume du pot... elle est fatiguée et elle veut se coucher... Alors, $j$ 'y dis : "OK, tu peux te coucher, mais après, essaye de prendre soin de moi »... Je ne voulais pas qu'elle s'en aille sans que j'aie mon « nanane »! (François, 23 ans)

\section{Discussion}

Cet article visait à décrire les différences de genre au sein des relations intimes chez les jeunes en situation d'itinérance. À partir d'une ADS, il a mis en évidence les points de convergence et de divergence dans la construction des relations intimes chez les jeunes femmes et les jeunes hommes rencontrés.

En ce qui concerne les points de convergence, les résultats révèlent que les conditions de vie difficiles de la situation d'itinérance exercent une influence notable sur les relations intimes de ces jeunes, ce constat s'accordant avec d'autres travaux de recherche (Lanzarini, 2000 ; Laporte et al., 2007 ; Osthus et Sewpaul, 2014). II ressort de l'analyse que les relations intimes chez ces jeunes sont difficiles à établir et à maintenir en raison de leurs conditions de vie précaires et instables, mais qu'elles sont considérées, de façon paradoxale, comme importantes pour répondre à leurs besoins de subsistance. En raison du poids de la survie, les jeunes sont amenés à se replier sur eux-mêmes plutôt que de s'ouvrir aux autres, cela engendrant ce que Jamoulle (2009) désigne comme de la "solitude affective". Les jeunes en situation d'itinérance se retrouvent alors déconnectés 
affectivement les uns des autres, et ce, avec l'impression qu'il n'est pas possible pour eux d'établir un réel contact intime avec autrui. Cette solitude affective participe à construire, chez les jeunes, un sentiment d'inquiétude, de menace et de danger par rapport à la situation d'itinérance, ce qui les pousse, paradoxalement, à rechercher des contacts intimes pour contrer ce sentiment d'isolement et de détresse psychologique. Les relations intimes sont alors conçues comme importantes pour s'organiser, se protéger et se valoriser dans le contexte de l'itinérance. De façon similaire aux travaux de Pichon (2007, p. 71), nous avons constaté que la précarité incite les personnes en situation d'itinérance à « construire des liens de sociabilité efficaces auprès des bénévoles, des professionnels et surtout auprès de ceux qui se trouvent dans la même situation ». Dans ce contexte de précarité et d'instabilité, les jeunes en situation d'itinérance sont amenés à façonner des relations intimes dans le but de combler leurs besoins matériels, affectifs et sécuritaires. Le fait de nouer des relations intimes peut donc être vu comme une stratégie de résistance commune pour contrer la précarité économique, l'instabilité résidentielle et les dangers associés aux conditions de vie de la situation d'itinérance.

Cette stratégie de résistance commune pourrait, de prime abord, faire croire que la précarité et l'instabilité de la situation d'itinérance annihilent toutes formes de rapports de pouvoir entre les genres et que les relations intimes se développent sur le partage égalitaire des ressources matérielles et économiques entre les jeunes femmes et les jeunes hommes pour assurer leur survie. Toutefois, l'ADS des expériences des jeunes montre qu'il n'en est rien. Bien au contraire, elle met en évidence que des rapports de pouvoir se cachent au cœur des relations intimes entre les jeunes femmes et les jeunes hommes en situation d'itinérance. En effet, l'itinérance constitue, chez les jeunes, un vecteur de reproduction des rôles de genre traditionnels qui exacerbe les inégalités de genre dans les relations intimes.

Dans un premier temps, les jeunes femmes conçoivent l'itinérance comme une expérience porteuse de menace et de dangerosité pour leur intégrité physique, mentale et sexuelle. Pour contrer les dangers associés à cette situation, elles développent des liens amoureux, affectifs et sexuels avec des partenaires intimes qui leur permettent d'assurer leur sécurité et leur protection. Ces résultats font écho aux travaux qui montrent que les partenaires intimes constituent une ressource économique et matérielle importante pour les jeunes femmes en situation d'itinérance (Kirst et al., 2009 ; Watson, 2013 ; Flynn et al., 2015). Néanmoins, la majorité des jeunes femmes rencontrées mentionnent avoir vécu de la violence de la part de leur partenaire intime dans le contexte de l'itinérance, un constat qui concorde avec d'autres travaux (Cauce et al., 2000 ; Watson, 2011). Comme Watson (2013), nous constatons que les jeunes femmes semblent se résigner à endurer des relations intimes marquées par la violence plutôt que de se retrouver isolées, sans ressources et sans protection, dans un contexte qu'elles considèrent comme menaçant et dangereux. Ce sentiment d'insécurité évoque le peu de ressources auxquelles les jeunes femmes ont accès en situation d'itinérance et témoigne de la nécessité, pour elles, d'établir des relations intimes pour répondre à leurs besoins de survie. L'acceptation de la violence dans leurs relations intimes illustre donc l'oppression à laquelle les jeunes femmes en situation d'itinérance sont soumises et les stratégies qu'elles doivent mobiliser pour tenter de contourner leur position sociale discriminée.

Dans notre étude (et cela correspond aux constats de Lanzarini, 2000 ; O'Grady et Gaetz, 2004 ; Jamoulle, 2009 ; Watson, 2011), plusieurs des jeunes femmes rencontrées disent mobiliser, à certains moments de leur vie en situation d'itinérance, la seule ressource dont elles croient pouvoir tirer profit, c'est-à-dire leur sexualité. L'omniprésence perçue de la violence en situation d'itinérance semble 
inciter ces jeunes femmes à adopter des pratiques prostitutionnelles, qui sont jugées par elles plus sécuritaires pour leur santé physique que la vente de drogues, comme l'a rapporté d'ailleurs l'étude d'O'Grady et Gaetz (2004). Les rapports de genre inégalitaires créés par la précarité et l'instabilité en situation d'itinérance conduisent ces jeunes femmes à objectiver à la fois leur sexualité et leurs partenaires intimes afin de tirer avantage de la situation privilégiée de certains hommes, donc sans en éprouver aucun désir. En accord avec les conclusions de Tabet (2004), nous pensons que les témoignages des participantes mettent en évidence l'influence du système patriarcal qui les confine aux rôles traditionnels de la féminité, comme la dépendance et l'assujettissement aux attentes sexuelles de leurs partenaires en échange de compensations économiques et matérielles nécessaires à leur survie. Ces jeunes femmes se retrouvent donc dans un rapport de négociation déséquilibré, puisque les biens qu'elles veulent obtenir sont nécessaires à leur survie, contrairement à leurs partenaires qui, eux, disposent déjà des ressources économiques et matérielles permettant d'assurer leur subsistance.

Pour leur part, certains jeunes hommes vivent la situation d'itinérance comme une expérience humiliante. Ces jeunes hommes indiquent que l'absence de ressources économiques et matérielles en situation d'itinérance fait qu'ils ont l'impression de ne pas correspondre aux rôles de genre traditionnels de la masculinité qui comprennent, entre autres, l'indépendance et la fonction de pourvoyeur. Ce sentiment de ne pas être conformes aux rôles de genre traditionnels suscite, chez ces jeunes hommes, l'impression d'être incapables de se présenter de façon séduisante et attirante à des partenaires intimes potentielles. Comme d'autres travaux l'ont déjà montré (Laporte et al., 2007 ; Kennedy et al., 2013), l'incapacité de ces jeunes hommes à pourvoir aux besoins de partenaires intimes potentielles les incite à rompre avec toutes formes de relations intimes, leur évitant ainsi de faire face à l'humiliation de dévoiler leurs conditions de vie précaires et instables. L'expérience d'un statut socio-économique précaire semble donc faire obstacle, chez certains jeunes hommes, à leur capacité d'entrer en contact avec des partenaires intimes, comme s'ils avaient l'impression de ne pas être à la hauteur des attentes sociales concernant la contribution financière et matérielle que doit fournir un partenaire masculin au sein d'une relation intime.

C'est l'investissement dans les activités criminelles qui donne accès, à certains jeunes hommes en situation d'itinérance, aux ressources économiques et matérielles qui leur procurent un statut privilégié. La capacité de ces jeunes hommes à accéder à ces ressources valide leur masculinité, dans la mesure où ils adhèrent aux rôles traditionnels de la masculinité, tels que l'indépendance et le rôle de pourvoyeur. Cette adhésion aux rôles traditionnels de la masculinité donne l'impression aux jeunes hommes qu'ils peuvent exiger de leurs partenaires des activités sexuelles contre de l'argent ou un hébergement (Lanzarini, 2000 ; Jamoulle, 2005 ; Laporte et al., 2007). Tout comme Tabet (2004), nous avançons l'idée que la catégorisation sociale du genre positionne les jeunes hommes dans un rapport de domination sur les jeunes femmes dans la mesure où ils peuvent s'investir dans des activités économiques dangereuses et, par conséquent, plus lucratives, ce qui leur donne accès à des ressources pouvant être négociées contre des relations sexuelles. Ce rapport de domination fait que ces jeunes hommes ont l'impression d'être socialement justifiés de concevoir les femmes comme des objets de satisfaction de leur plaisir sexuel, en ayant peu d'égards pour le désir de ces dernières. Cette position sociale de domination et le pouvoir de séduction que les jeunes hommes disent en retirer semblent donc renforcer leur identité masculine et, par le fait même, valider leur adhésion aux rôles de genre traditionnels de la masculinité. 


\section{Conclusion}

Cette étude montre l'existence de différences de genre dans la construction des relations intimes chez les jeunes en situation d'itinérance. L'ADS illustre que la précarité et l'instabilité de la situation d'itinérance tendent à créer des relations intimes fondées sur des rôles de genre traditionnels qui engendrent, par le fait même, des rapports inégalitaires entre les jeunes femmes et les jeunes hommes. Ces rapports inégalitaires ne sont toutefois pas le fruit du hasard. Ils sont façonnés par un système patriarcal qui exacerbe, dans le contexte de la précarité socio-économique, les rapports de domination et d'oppression. Dans un contexte social où les relations intimes contemporaines sont considérées comme un mécanisme de construction identitaire et de définition de soi (Giddens, 1992 ; Beck et Beck-Gernsheim, 1995 ; Bauman, 2004), les jeunes en situation d'itinérance semblent mobiliser les relations amoureuses et sexuelles comme des ressources pour améliorer leurs conditions de vie. Comme les relations intimes restent structurées par le système patriarcal (Tabet, 2004), les jeunes femmes en situation d'itinérance sont poussées à les instrumentaliser pour assurer leur subsistance, tandis que les jeunes hommes en situation d'itinérance, en raison de leur position sociale privilégiée, sont soutenus dans l'instrumentalisation de leurs partenaires féminines pour accéder à la sexualité et renforcer leur masculinité. L'analyse des relations intimes en situation d'itinérance que présente cet article met en lumière l'importance de travailler à la sensibilisation et à l'éducation des jeunes dans une visée de déconstruction des rapports de genre inégalitaires, tout particulièrement chez ceux qui se retrouvent en situation de précarité économique et d'instabilité résidentielle. La présente analyse ne permet toutefois pas de capter l'intersection, dans la construction des relations intimes chez les jeunes, des catégorisations sociales telles que la race, l'orientation sexuelle et l'identité de genre. Le cadre théorique de l'intersectionnalité semble être une avenue pertinente pour guider de tels travaux.

\section{REMERCIEMENTS}

Les auteur.es remercient les participant.es pour leur générosité, Josiane Maheu pour son aide apportée à la conceptualisation de l'analyse différenciée selon les sexes, ainsi que Marie-Andrée Provencher, Michel Martel, Marie-Hélène Proulx et Jean-François Truchon pour leur contribution à des phases antérieures de la recherche. Cette recherche a été financée par le Conseil de recherches en sciences humaines.

CÔTÉ, Philippe-Benoit, Ph. D., professeur Département de sexologie

Université du Québec à Montréal

FLYNN, Catherine, Ph. D., professeure associée Département de psychosociologie et de travail social

Université du Québec à Rimouski

BLAIS, Martin, Ph. D., professeur Département de sexologie

Université du Québec à Montréal 
MANSEAU, Hélène, Ph. D., professeure Département de sexologie Université du Québec à Montréal

FOURNIER, Émilie, M.A. Département de sexologie Université du Québec à Montréal

\section{RÉFÉRENCES}

Bauman, Z. (2004). L'amour liquide. De la fragilité des liens entre les hommes, Rodez, Le Rouergue/Chambon.

Beck, U., et E. Beck-Gernsheim (1995). The Normal Chaos of Love, Cambridge, Mass., Polity Press.

Bellot, C. (2003). "Les jeunes de la rue: disparition ou retour des enjeux de classe ? ", Lien social et Politiques, $\mathrm{n}^{\circ}$ 49, p. 173-182.

Blais, M., P.-B. Côté, H. Manseau, M. Martel et M.-A. Provencher (2012). "Love among the homeless: A portrait of romantic and couple relationships among street-involved young adults in Montreal », Journal of Youth Studies, vol. 15, n 4, p. 403-420.

Castel, R. (1998). Les sorties de la toxicomanie, Fribourg, Éditions universitaires de Fribourg.

Cauce, A. M., M. Paradise, J. A. Ginzler, L. Embry, C. J. Morgan, Y. Lohr et J. Theofelis (2000). « The characteristics and mental health of homeless adolescents: Age and gender differences ", Journal of Emotional and Behavioral Disorders, vol. 8, n 4, p. 230-239.

Côté, P.-B., M. Blais, C. Bellot et H. Manseau (2013). " Des expériences affectives et sexuelles en situation de rue », Criminologie, vol. 46, nº 2, p. 243-262.

Côté, P.-B., M. Blais, C. Bellot et H. Manseau (2016). "Les relations affectives et sexuelles des jeunes en situation de rue : entre investissement et désinvestissement ", Sociétés et jeunesses en difficulté, $\mathrm{n}^{\circ} 17$, p. 1-19.

Flynn, C., D. Damant et G. Lessard (2015). «Le projet Dauphine : laisser la parole aux jeunes femmes de la rue et agir ensemble pour lutter contre la violence structurelle par l'entremise de la recherche-action participative ", Recherches féministes, vol. 28, n², p. 53-79.

Giddens, A. (1992). La transformation de l'intimité. Sexualité, amour et érotisme dans les sociétés modernes, Rodez, Le Rouergue/Chambon.

Gwadz, M. V., D. Nish, N. R. Leonard et S. M. Strauss (2007). "Gender differences in traumatic events and rates of post-traumatic stress disorder among homeless youth ", Journal of Adolescence, vol. $30, n^{\circ} 1$, p. 117-129. 
Huey, L., et E. Berndt (2008). “'You've gotta learn how to play the game': Homeless women's use of gender performance as a tool for preventing victimization », The Sociological Review, vol. 56, $\mathrm{n}^{\circ} 2$, p. 177-194.

Jamoulle, P. (2005). Des hommes sur le fil. La construction de l'identité masculine en milieux précaires, Paris, La Découverte.

Jamoulle, P. (2009). Fragments d'intime. Amours, corps et solitudes aux marges urbaines, Paris, La Découverte.

Kennedy, D. P., R. A. Brown, D. Golinelli, S. L. Wenzel, J. S. Tucker et S. R. Wertheimer (2013). «Masculinity and HIV risk among homeless men in Los Angeles », Psychology of Men \& Masculinity, vol. 14, $\mathrm{n}^{\circ}$ 2, p. 156-167.

Kirst, M. J., P. Erickson et C. Strike (2009). «Poly-substance use among male and female street youth in Toronto, Canada », International Journal of Social Inquiry, vol. 2, n² 2, p. 123-139.

Lanzarini, C. (2000). Survivre dans le monde sous-prolétaire, Paris, Presses universitaires de France.

Laperrière, A. (1997). "La théorisation ancrée (grounded theory): démarche analytique et comparaison avec d'autres approches apparentées », dans J. Poupart, J.-P. Deslauriers, L.-H. Groulx, A. Laperrière, R. Mayer et A. Pires, La recherche qualitative. Enjeux épistémologiques et méthodologiques, Montréal, Gaëtan Morin Éditeur, p. 309-333.

Laporte, A., E. Le Ménier, N. Oppenchaim, D. Pourette et S. Carpentier (2007). Survivre ou faire l'amour? La pluralité des expériences affectives et sexuelles de personnes sans domicile fixe, Paris, Rapport à l'INPES.

Mahalik, J. R., B. D. Locke, L. H. Ludlow, M. A. Diemer, R. P. Scott, M. Gottfried et G. Freitas (2003). "Development of the conformity to masculine norms inventory », Psychology of Men \& Masculinity, vol. $4, \mathrm{n}^{\circ} 1$, p. 3-25.

Mahalik, J. R., E. B. Morray, A. Coonerty-Femiano, L. H. Ludlow, S. M. Slattery et A. Smiler (2005). «Development of the conformity to feminine norms inventory ", Sex Roles, vol. 52, n 7-8, p. 417435.

Massé, H., M. Laberge et G. Massé (2002). « L'analyse différenciée selon les sexes au gouvernement du Québec : vers une mobilisation interne et des alliances stratégiques pour l'égalité », Lien social et Politiques, $\mathrm{n}^{\circ} 47$, p. 43-54.

Ministère de la Santé et des Services sociaux (2011). Analyse différenciée selon les sexes dans le secteur de la santé et des services sociaux - Guide-mémoire, Québec, Gouvernement du Québec.

O'Grady, B., et S. Gaetz (2004). «Homelessness, gender and subsistence: The case of Toronto street youth », Journal of Youth Studies, vol. 7, n 4, p. 397-416.

Osthus, I. S., et V. Sewpaul (2014). «Gender, power and sexuality among youth on the streets of 
Durban: Socio-economic realities », International Social Work, vol. 57, n 4, p. 326-337.

Pichon, P. (2007). Vivre sans domicile fixe : l'épreuve de l'habitat précaire, Montreuil, Aux lieux d'être.

Roy, É., N. Haley, P. Leclerc, N. Lemire, J.-F. Boivin, J.-Y. Frappier et C. Classens (2000). "Prevalence of HIV infection and risk behaviours among Montreal street youth", International Journal of STD \& AIDS, vol. 11, nº 4, p. 241-247.

Secrétariat à la condition féminine (2007). L'analyse différenciée selon les sexes dans les pratiques gouvernementales et dans celles des instances locales et régionales - Guide synthèse, Québec, Ministère de la Culture, des Communications et de la Condition féminine.

Stablein, T. (2011). "Helping friends and the homeless milieu: Social capital and the utility of street peers ", Journal of Contemporary Ethnography, vol. 40, n 3 , p. 290-317.

Tabet, P. (2004). La grande arnaque. Sexualité des femmes et échange économico-sexuel, Paris, L'Harmattan.

Tesch, R. (1990). Qualitative research: Analysis types and software tools, New York, The Falmer Press.

Tolman, D. L., M. I. Striepe et T. Harmon (2003). "Gender matters: Constructing a model of adolescent sexual health ", Journal of Sex Research, vol. 40, n 1, p. 4-12.

Walls, N. E. et S. Bell (2011). "Correlates of engaging in survival sex among homeless youth and young adults », Journal of Sex Research, vol. 48, n 5, p. 423-436.

Watson, J. (2011). "Understanding survival sex: Young women, homelessness and intimate relationships », Journal of Youth Studies, vol. 14, n 6, p. 639-655.

Watson, J. (2013). « Homelessness, gender-based violence and social capital: The function of sexual alliances », Parity, vol. 26, n 10, p. 13-14. 